\title{
A SET THEORY BASED ON A CERTAIN 3-VALUED LOGIC
}

\author{
TH. SKOLEM
}

\section{Introduction.}

In set theory one has to do with more or less extensive forms of the so-called axiom of comprehension which can be written

$$
(E y)(x)(x \in y \leftrightarrow \Phi(x)),
$$

where $\Phi(x)$ is some propositional function in which the variable $x$, but not $y$, occurs. It is well known that a complete axiom of this kind leads to contradictions. It is worth noticing that this is so even if no quantifiers occur in $\Phi(x)$. Indeed, letting for example $\Phi(x)$ denote the very simple propositional function $x \bar{E} x$, the equivalence $x \in y \leftrightarrow x \bar{\in} x$ assumed valid for all $x$ leads to the contradiction $y \in y \leftrightarrow y \bar{\epsilon} y$ which is the famous antinomy of Russell.

It is the aim of this paper to show that the situation is quite different if we take into account a suitable 3-valued logic. In [1] I showed that the antinomies could be avoided in the case of quantifierfree $\Phi$, if we applied an infinitely many-valued logic due to Eukasiewicz. However, it turns out that already a certain 3-valued logic is sufficient for attaining that. In this logic no further operation concerning propositions are used than just negation, conjunction and disjunction. I shall first give a brief explanation of this logic and then prove that it is possible to construct a domain of individuals with a binary relation $\varepsilon$ therein such that not only the axiom of comprehension but also the axiom of extensionality are fulfilled.

\section{1.}

Let $p, q, r, \ldots$ be propositional variables, taking three values $0, \frac{1}{2}, 1$. We may interprete 0 as false, 1 as true and $\frac{1}{2}$ as something in the middle between true and false, say "possible" or "undetermined". These 3 values are called "truthvalues". We assume 3 operations, a unary one, negation, and two binary ones, called conjunction and disjunction. 
The truthvalues of the result of an operation is determined by the truthvalues of the operands according to the following tables:

Negation of $p$

written $\urcorner p$

\begin{tabular}{c|c|c|c|}
$p$ & 0 & $\frac{1}{2}$ & 1 \\
\hline $7 p$ & 1 & $\frac{1}{2}$ & 0
\end{tabular}

Conjunction of $p$ and $q$ written $p \& q$

\begin{tabular}{c|c|c|c|}
$p \backslash$ & 0 & $\frac{1}{2}$ & 1 \\
\hline 0 & 0 & 0 & 0 \\
\hline$\frac{1}{2}$ & 0 & $\frac{1}{2}$ & $\frac{1}{2}$ \\
\hline 1 & 0 & $\frac{1}{2}$ & 1 \\
\hline
\end{tabular}

Disjunction of $p$ and $q$ written $p \vee q$

\begin{tabular}{|c|c|c|c|}
\hline$p q^{q}$ & 0 & $\frac{1}{2}$ & 1 \\
\hline 0 & 0 & $\frac{1}{2}$ & 1 \\
\hline$\frac{1}{2}$ & $\frac{1}{2}$ & $\frac{1}{2}$ & 1 \\
\hline 1 & 1 & 1 & 1 \\
\hline
\end{tabular}

Letting the letters stand for the truthvalues we may replace $7 p$ by $1-p$, $p \& q$ by $\min (p, q)$ and $p \vee q$ by $\max (p, q)$.

It is natural to say that two functions $f$ and $g$ are identical and write $f=g$, if they always take the same values. We have for example the equations

$$
\urcorner\urcorner p=p, \quad\urcorner(p \& q)=\urcorner p \vee\urcorner q, \quad\urcorner(p \vee q)=\urcorner p \&\urcorner q
$$

and therefore also

$$
p \& q=\urcorner(\urcorner p \vee\urcorner q), \quad p \vee q=\urcorner(\urcorner p \&\urcorner q)
$$

so that conjunction can be defined by negation and disjunction or inversely disjunction by negation and conjunction. All this is just as in ordinary 2-valued logic. There are some differences however. Thus the tertium non datur namely

$$
p \vee\urcorner p=1
$$

is not valid here, the value $\frac{1}{2}$ of $p$ yielding $\left.p \vee\right\urcorner p=\frac{1}{2}$. Similarly $\left.p \&\right\urcorner p=0$ in 2-valued logic, whereas here $p \&\urcorner p=\frac{1}{2}$ when $p=\frac{1}{2}$.

A thorough treatment of this 3-valued propositional logic might be of interest, but in the following very little knowledge of it is needed. I will therefore only make a few remarks. Apart from the 3 constants $0, \frac{1}{2}, 1$ there are 8 further functions of one variable $p$. These 11 functions are shown in the table below, where the values of the argument $p$ stand in the first row and the values of the functions in the $2^{\text {nd }}$ to $12^{\text {th }}$ rows.

The reader will easily convince himself that no further function of $p$ is constructible by use of the three fundamental operations.

If the equation $f(p)=p$ takes place for a certain value $p$ of $p$, we say that $p$ is a fixed point for $f(p)$. Looking at the table above we see that all of the 11 functions, possess fixed points. Apart from the constant 0 
Choice of

\begin{tabular}{l|c|c|c|c} 
& 0 & $\frac{1}{2}$ & 1 & fixed point \\
constant 0 & 0 & 0 & 0 & 0 \\
$p \&\urcorner p$ & 0 & $\frac{1}{2}$ & 0 & 0 \\
$\frac{1}{2} \& p$ & 0 & $\frac{1}{2}$ & $\frac{1}{2}$ & 0 \\
$\left.\frac{1}{2} \&\right\urcorner p$ & $\frac{1}{2}$ & $\frac{1}{2}$ & 0 & $\frac{1}{2}$ \\
$p$ & 0 & $\frac{1}{2}$ & 1 & $\frac{1}{2}$ \\
constant $\frac{1}{2}$ & $\frac{1}{2}$ & $\frac{1}{2}$ & $\frac{1}{2}$ & $\frac{1}{2}$ \\
\urcorner $p$ & 1 & $\frac{1}{2}$ & 0 & $\frac{1}{2}$ \\
$\frac{1}{2} \vee p$ & $\frac{1}{2}$ & $\frac{1}{2}$ & 1 & 1 \\
$\left.\frac{1}{2} \vee\right\urcorner p$ & 1 & $\frac{1}{2}$ & $\frac{1}{2}$ & $\frac{1}{2}$ \\
$p \vee\urcorner p$ & 1 & $\frac{1}{2}$ & 1 & 1 \\
constant 1 & 1 & 1 & 1 & 1
\end{tabular}

with 0 as fixed point and the constant 1 with 1 as fixed point every function has $\frac{1}{2}$ as a fixed point. One observes that the fixed points, can be chosen in more ways, but it is of some importance in the sequel to have a unique choice of them. Such a choice is indicated in the table above.

As a preparation for the proof of the more far-reaching theorem in section 2 , I show in this section that we may determine the values of $\varepsilon(x, y)$ for the diverse pairs $x, y$ in the domain of 11 objects $0,1,2,3,4,5$, $6,7,8,9,10$ such that the axiom of comprehension is satisfied for propositional functions $\Phi(x)$ containing neither quantifiers nor free variables different from $x$ (parameters). Indeed an example is given by the following table:

\begin{tabular}{r|l|l|l|l|l|l|l|l|l|l|l|}
$\varepsilon$ & 0 & 1 & 2 & 3 & 4 & 5 & 6 & 7 & 8 & 9 & 10 \\
\hline 0 & 0 & 0 & 0 & $\frac{1}{2}$ & 0 & $\frac{1}{2}$ & 1 & $\frac{1}{2}$ & 1 & 1 & 1 \\
1 & 0 & 0 & 0 & $\frac{1}{2}$ & 0 & $\frac{1}{2}$ & 1 & $\frac{1}{2}$ & 1 & 1 & 1 \\
2 & 0 & 0 & 0 & $\frac{1}{2}$ & 0 & $\frac{1}{2}$ & 1 & $\frac{1}{2}$ & 1 & 1 & 1 \\
3 & 0 & $\frac{1}{2}$ & $\frac{1}{2}$ & $\frac{1}{2}$ & $\frac{1}{2}$ & $\frac{1}{2}$ & $\frac{1}{2}$ & $\frac{1}{2}$ & $\frac{1}{2}$ & $\frac{1}{2}$ & 1 \\
4 & 0 & $\frac{1}{2}$ & $\frac{1}{2}$ & $\frac{1}{2}$ & $\frac{1}{2}$ & $\frac{1}{2}$ & $\frac{1}{2}$ & $\frac{1}{2}$ & $\frac{1}{2}$ & $\frac{1}{2}$ & 1 \\
5 & 0 & $\frac{1}{2}$ & $\frac{1}{2}$ & $\frac{1}{2}$ & $\frac{1}{2}$ & $\frac{1}{2}$ & $\frac{1}{2}$ & $\frac{1}{2}$ & $\frac{1}{2}$ & $\frac{1}{2}$ & 1 \\
6 & 0 & $\frac{1}{2}$ & $\frac{1}{2}$ & $\frac{1}{2}$ & $\frac{1}{2}$ & $\frac{1}{2}$ & $\frac{1}{2}$ & $\frac{1}{2}$ & $\frac{1}{2}$ & $\frac{1}{2}$ & 1 \\
7 & 0 & 0 & $\frac{1}{2}$ & 0 & 1 & $\frac{1}{2}$ & 0 & 1 & $\frac{1}{2}$ & 1 & 1 \\
8 & 0 & $\frac{1}{2}$ & $\frac{1}{2}$ & $\frac{1}{2}$ & $\frac{1}{2}$ & $\frac{1}{2}$ & $\frac{1}{2}$ & $\frac{1}{2}$ & $\frac{1}{2}$ & $\frac{1}{2}$ & 1 \\
9 & 0 & 0 & $\frac{1}{2}$ & 0 & 1 & $\frac{1}{2}$ & 0 & 1 & $\frac{1}{2}$ & 1 & 1 \\
10 & 0 & 0 & $\frac{1}{2}$ & 0 & 1 & $\frac{1}{2}$ & 0 & 1 & $\frac{1}{2}$ & 1 & 1
\end{tabular}

$$
\begin{aligned}
\varepsilon(x, 0) & =0 \\
\varepsilon(x, 1) & =\min (\varepsilon(x, x), 1-\varepsilon(x, x)) \\
\varepsilon(x, 2) & =\min \left(\frac{1}{2}, \varepsilon(x, x)\right) \\
\varepsilon(x, 3) & =\min \left(\frac{1}{2}, 1-\varepsilon(x, x)\right) \\
\varepsilon(x, 4) & =\varepsilon(x, x) \\
\varepsilon(x, 5) & =\frac{1}{2} \\
\varepsilon(x, 6) & =1-\varepsilon(x, x) \\
\varepsilon(x, 7) & =\max \left(\frac{1}{2}, \varepsilon(x, x)\right) \\
\varepsilon(x, 8) & =\max \left(\frac{1}{2}, 1-\varepsilon(x, x)\right) \\
\varepsilon(x, 9) & =\max (\varepsilon(x, x), 1-\varepsilon(x, x)) \\
\varepsilon(x, 10) & =1
\end{aligned}
$$

Math. Scand. $8-9$ 
One observes that two different individuals do not have the same elements, that is, if $y$ and $z$ are two different individuals from the sequence $0,1, \ldots, 10$ we dont have $\varepsilon(x, y)=\varepsilon(x, z)$ for all $x$. Therefore, also the axiom of extensionality is fulfilled. Curiously enough however, different elements $x$ and $y$ are sometimes elements of the same sets, that is, $\varepsilon(x, z)=\varepsilon(y, z)$ for all $z$.

2.

I consider now propositional functions which can be constructed by use of the 3 operations from the fundamental binary function $\varepsilon(x, y)$. The reader may interprete $\varepsilon(x, y)$ as the membership relation " $x$ is element of $y$ ". For each pair of individuals $x$ and $y$ the truthvalue of $\varepsilon(x, y)$ may be $0, \frac{1}{2}$ or 1 . A general propositional function can then be written

$$
U\left(x, x_{1}, x_{2}, \ldots, x_{n}\right),
$$

where $U$ is built up by the 3 operations from atomic statements $\varepsilon(x, x)$, $\varepsilon\left(x, x_{r}\right), \varepsilon\left(x_{r}, x\right), \varepsilon\left(x_{r}, x_{s}\right), r, s=1,2, \ldots, n$ independently.

I shall now show that it is possible to construct a domain of individuals together with the relation $\varepsilon$ between them such that the following comprehension statement is valid:

Given for arbitrary $n$ an arbitrary propositional $(n+1)$-ary function $U$ together with $n$ arbitrary individuals $x_{1}, x_{2}, \ldots, x_{n}$. Then there exists an individual $y$ such that for every $x$ the truthvalue of $\varepsilon(x, y)$ is just equal to the value of $U\left(x, x_{1}, \ldots, x_{n}\right)$. Of course $y$ varies according to the choice of $n, U$, and the individuals $x_{1}, \ldots, x_{n}$.

In order to prove this I make use of an enumeration of the propositional functions $U$. Let the $n^{\text {th }}$ of them be written $U_{n}\left(x, x_{1}, x_{2}, \ldots, x_{g(n)}\right)$. The individuals of the domain shall be constructed from an original one, 0 , by use of the following procedure. To every $U_{n}\left(x, x_{1}, \ldots, x_{g(n)}\right)$ we associate the expression $f_{n}\left(x_{1}, \ldots, x_{g(n)}\right)$. Then, when $n$ is an arbitrary positive integer, $x_{1}, \ldots, x_{g(n)}$ arbitrary previously constructed individuals, $f_{n}\left(x_{1}, \ldots, x_{g(n)}\right)$ shall be a new individual. In case $g(n)=0$, I write simply $f_{n}$. Now, I shall prove by induction that it is possible to determine successively the truthvalues of $\varepsilon(u, v)$ for the diverse pairs $u, v$ such that for $v=f_{n}\left(x_{1}, \ldots, x_{g(n)}\right)$ the value of $\varepsilon(x, v)$ is, for all $x$, just the same as the value of $U_{n}\left(x, x_{1}, \ldots, x_{g(n)}\right)$. This inductive proof is in so far a little complicated as it contains another proof by induction as a part of itself.

I introduce the notion "height" of an individual in the following way. The original individual is said to have the height 0 . If $h$ is the maximal height of $x_{1}, \ldots, x_{g(n)}$, then $h+1$ shall be the height of the individual $f_{n}\left(x_{1}, \ldots, x_{g(n)}\right)$. For $g(n)=0$ we take $f_{n}$ to be of height 1 . 
Now $\varepsilon(x, 0)$ is chosen equal 0 for all individuals $x$. Then $\varepsilon(u, v)$ is determined for all $u, v$ of height 0 , namely $u=0, v=0$. Letting $U_{0}(x)$ denote the propositional constant 0 , we have $\varepsilon(x, 0)=U_{0}(x)$ for all $x$, hence à fortiori $\varepsilon(x, 0)=U_{0}(x)$ for all $x$ of height 0 , namely the individual 0 . Let us now assume that we have been able to determine the value of $\varepsilon(u, v)$ for all $u, v$ of height $\leqq h$ in such a way that, if $v=f_{l}\left(x_{1}, \ldots, x_{g(l)}\right)$, then

$$
\varepsilon(u, v)=U_{l}\left(u, x_{1}, \ldots, x_{g(l)}\right) .
$$

Then I show that this determination can be continued so that the same equation holds for all pairs $u, v$, where $u$ and $v$ have heights $₹ h+1$.

Let us first consider $\varepsilon(x, y)$, where $x$ is still of the height $₹ h$, whereas $y$ has height $h+1$. We have then $y=f_{n}\left(x_{1}, \ldots, x_{g(n)}\right)$, where the heights of $x_{1}, \ldots, x_{g(n)}$ are all $₹ h$. According to supposition all atomic propositions $\varepsilon(x, x), \varepsilon\left(x, x_{r}\right), \varepsilon\left(x_{r}, x\right), \varepsilon\left(x_{r}, x_{s}\right)$ from which $U_{n}\left(x, x_{1}, \ldots, x_{g(n)}\right)$ is built up have got values in accordance with the statement of comprehension. Then the value of $U_{n}$ can be computed and determined as the value of $\varepsilon(x, y)$. Now let $x$ be of height $h+1$. Then I first prove, again by induction, that the values of $\varepsilon(x, z)$ for the diverse $z$ of height $\sum h+1$ can be preliminarely determined as functions of $p=\varepsilon(x, x)$ in such a way that all equations of comprehension become identities in $p$. This is certainly true when $z$ is of height 0 , because $\varepsilon(x, 0)=0$ and $U_{0}(x)=0$ so that $p$ does not occur at all. We assume this determination carried out for $z$ of

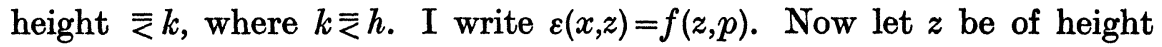
$k+1$ and $=f_{m}\left(x_{1}, \ldots, x_{g(m)}\right)$. Then according to supposition the atomic propositions $\varepsilon\left(x, x_{r}\right)$ in $U_{m}\left(x, x_{1}, \ldots, x_{g(m)}\right)$ are determined as functions of $p$ while all $\varepsilon\left(x_{r}, x\right)$ have determined values according to the procedure already explained because $x_{r}$ is of height $h$ at most and $x$ of height $h+1$. Further, the values of all $\varepsilon\left(x_{r}, x_{s}\right)$ are known. Then $U_{m}$ can be computed as a function of $p$ and this function can be put equal to $\varepsilon(x, z)$. Thus the determination of the $\varepsilon(x, z)$ as functions of $p$ in accordance with the comprehension principle can be extended to the $z$ of height $k+1$ and therefore to all $z$ of height $\leqq h+1$. In particular, in the case $z=x$ we get $p=\varepsilon(x, x)=f(x, p)$. Now the equation $p=f(x, p)$ has a fixed point. We may choose that as indicated earlier. Then $p$ is determined so that the value of $\varepsilon(x, x)$ is equal to $U_{n}\left(x, x_{1}, \ldots, x_{g(n)}\right)$ if $x=f_{n}\left(x_{1}, \ldots, x_{g(n)}\right)$. By insertion of this value of $p$ in the diverse $f(z, p)$ we find values of the diverse $\varepsilon(x, z)$ so that the statement of comprehension becomes true for all these $z$. Finally, if also $y$ is an individual of height $h+1$, different from $x$, and say $y=f_{r}\left(x_{1}, \ldots, x_{g(r)}\right)$, then we now know the values of all atomic statements in $U_{r}\left(x, x_{1}, \ldots, x_{g(r)}\right)$. Thus we have only to compute the value of $U_{r}$ and take it as a value of $\varepsilon(x, y)$. Then the comprehen- 
sion requirement is fulfilled for all individuals up to height $h+1$. Hence it follows that the construction can be continued to individuals of arbitrary heights in such a way that we get a domain in which the axiom of comprehension is satisfied.

3.

The axiom of extensionality says that if $\varepsilon(u, x)=\varepsilon(u, y)$ for all $u$, then $\varepsilon(x, v)=\varepsilon(y, v)$ for all $v$. I shall prove that also this axiom is fulfilled in the domain constructed in section 2.

Let $\varepsilon(u, x)=\varepsilon(u, \xi)$ for all $u$. I assert that $\varepsilon(x, x)=\varepsilon(\xi, \xi)$. Indeed, let

Then

$$
x=f_{n}\left(x_{1}, \ldots, x_{g(n)}\right), \quad \xi=f_{v}\left(\xi_{1}, \ldots, \xi_{g(v)}\right) .
$$

$$
\varepsilon(u, x)=U_{n}\left(u, x_{1}, \ldots, x_{g(n)}\right), \quad \varepsilon(u, \xi)=U_{v}\left(u, \xi_{1}, \ldots, \xi_{g(v)}\right) .
$$

Here $U_{n}\left(u, x_{1}, \ldots, x_{g(n)}\right)$ is a function of $\varepsilon(u, u)$ with coefficients depending on the other atomic propositions in $U_{n}$. Similarly for $U_{v}$. These coefficients must be such that we get in both cases the same function of $\varepsilon(u, u)$, say $F(\varepsilon(u, u))$. Indeed, if we had two different functions $F_{1}(\varepsilon(u, u))$ and $F_{2}(\varepsilon(u, u))$, we could by suitable choice of the value of $\varepsilon(u, u)$ get $F_{1} \neq F_{2}$, that is, $\varepsilon(u, x) \neq \varepsilon(u, \xi)$. Hence we may write

whence

$$
\varepsilon(u, x)=F(\varepsilon(u, u)), \quad \varepsilon(u, \xi)=F(\varepsilon(u, u)),
$$

$$
\varepsilon(x, x)=F(\varepsilon(x, x)), \quad \varepsilon(\xi, \xi)=F(\varepsilon(\xi, \xi)) .
$$

Because of the assumed unique choice of fixed points, $\varepsilon(x, x)=\varepsilon(\xi, \xi)$.

Now let $y=f_{n}\left(x_{1}, \ldots, x_{g(n)}\right)$ be of height $h+1$ assuming that we already know that $\varepsilon(x, v)=\varepsilon(\xi, v)$ is correct for all $v$ of height $\bar{k}$. Now

$$
\varepsilon(x, y)=U_{n}\left(x, x_{1}, \ldots, x_{g(n)}\right), \quad \varepsilon(\xi, y)=U_{n}\left(\xi, x_{1}, \ldots, x_{g(n)}\right) .
$$

Here the atomic propositions building up $U_{n}\left(x, x_{1}, \ldots, x_{g(n)}\right)$ namely $\varepsilon(x, x), \varepsilon\left(x_{r}, x\right), \varepsilon\left(x, x_{r}\right), \varepsilon\left(x_{r}, x_{s}\right)$ are equal those building up

$$
U_{n}\left(\xi, x_{1}, \ldots, x_{g(n)}\right)
$$

namely $\varepsilon(\xi, \xi), \varepsilon\left(x_{r}, \xi\right), \varepsilon\left(\xi, x_{r}\right)$ (this according to the hypothesis of induction), $\varepsilon\left(x_{r}, x_{s}\right)$, respectively so that one obtains

$$
\varepsilon(x, y)=\varepsilon(\xi, y) .
$$

Thus this equation is generally correct so that the axiom of extensionality is valid in the constructed domain of individuals.

By the way we may prove that the domain $D$ just constructed does not possess the strange property we encountered in the domain of 11 
objects mentioned in section 1, namely that two different objects $x$ and $y$ existed such that $\varepsilon(x, z)=\varepsilon(y, z)$ for all $z$. Indeed, let $x$ and $y$ be two different individuals in $D$. Then an individual $z$ exists such that $\varepsilon(z, x) \neq \varepsilon(z, y)$. One of the propositional functions $U\left(x, x_{1}, \ldots\right)$ has the simple form

$$
\varepsilon\left(x_{1}, x\right) \text {. }
$$

Now, for every individual $x_{1}$ there exists an individual $u$ such that for all $t$

Choosing $z$ for $x_{1}$ we get

$$
\varepsilon(t, u)=\varepsilon\left(x_{1}, t\right) \text {. }
$$

$$
\varepsilon(t, u)=\varepsilon(z, t)
$$

for all $t$ and therefore in particular

$$
\varepsilon(x, u)=\varepsilon(z, x), \quad \varepsilon(y, u)=\varepsilon(z, y) .
$$

Hence, because of $\varepsilon(z, x) \neq \varepsilon(z, y)$,

$$
\varepsilon(x, u) \neq \varepsilon(y, u) .
$$

4.

For the development of a set theory which could be sufficient for the foundation of mathematics it would of course be necessary to extend the axiom of comprehension to propositional functions with quantifiers. By the way, the use of quantifiers means the same as the use of minimum and maximum extended over arbitrary sets. We may distinguish between absolute and relative quantification. The relative one means that we take the minimum or maximum of all values of a propositional function $A(x)$ just for those $x$ for which a function $B(x)$ has the value 1 . Some time ago, I found that it would scarcely be difficult to develop mathematics, if we were allowed to use relative quantification as well as the absolute. However, it turns out that then our theory would become inconsistent. This can be proved in the following way.

Let

$$
\min _{x}(A(x) \mid B(x))
$$

denote the minimum of $A(x)$ for all $x$ for which $B(x)=1$. Let on the other hand an arrow $\rightarrow$ indicate the implication in the 3-valued logic according to Łukasiewicz. The operation $\rightarrow$ is defined by requiring the value of $A \rightarrow B$ to be

$$
\min (1,1-\text { the value of } A+\text { the value of } B) .
$$

Then, putting $\overline{A(x)}=1-A(x), \overline{B(x)}=1-B(x)$, we prove that if 


$$
\begin{array}{cr}
P=\min \left(\min _{x}(B(x) \mid A(x)), \min _{x}(\bar{A}(x) \mid \bar{B}(x))\right), \\
P=Q .
\end{array}
$$

then $P=Q$.

Let $Q$ have the value 0 . Now

so that

$$
\begin{aligned}
Q & =\min _{x}(\min (1,1-A(x)+B(x))) \\
& =\min \left(1, \min _{x}(1-A(x)+B(x))\right)
\end{aligned}
$$

for some $x$, whence $A(x)=1, B(x)=0$. This yields $P=0$. Let inversely $P=0$. Then there is at least one $x$ such that $A(x)=1$ and $B(x)=0$. For such an $x$ we get $1-A(x)+B(x)=0$, whence $Q=0$.

Now let $Q=\frac{1}{2}$. Then always $1-A(x)+B(x) \geqq \frac{1}{2}$, with equality holding for at least one $x$. Hence $A(x)=1$ implies $B(x)$ is $\geqq \frac{1}{2}$, and $B(x)=0$ implies $A(x) \leqq \frac{1}{2}$. This yields $P \geqq \frac{1}{2}$. For an $x$ such that $1-A(x)+B(x)=\frac{1}{2}$ we must have $A(x)=1, B(x)=\frac{1}{2}$ or $A(x)=\frac{1}{2}, B(x)=0$ so that $P=\frac{1}{2}$. Let inversely $P=\frac{1}{2}$. Then for $B(x)=0$ always $A(x) \leqq \frac{1}{2}$, and hence

$$
1-A(x)+B(x) \geqq \frac{1}{2}
$$

for all $x$. There is an $x$ such that $A(x)=1, B(x)=\frac{1}{2}$ or $B(x)=0, A(x)=\frac{1}{2}$. In both cases $1-A(x)+B(x)=\frac{1}{2}$. Thus $Q=\frac{1}{2}$.

Finally let $Q=1$. Then $A(x) \leqq B(x)$ for all $x$ so that also $\bar{B}(x) \leqq \bar{A}(x)$ for all $x$. This yields $P=1$. Let inversely $P=1$. Then $B(x)=1$ if $A(x)=1$ and $A(x)=0$ if $B(x)=0$. This means that $A(x) \leqq B(x)$ in all cases, whence $Q=1$.

The result is, therefore, that by taking into account relative quantification, we may construct such propositional functions as

$$
\min _{x}(A(x) \rightarrow B(x)),
$$

where the arrow denotes Łukasiewicz's implication.

According to the axiom of comprehension a set $m$ exists such that for all $z$

$$
\varepsilon(z, m)=\varepsilon(x, x) .
$$

Here $m$ depends on $x$, or in other words, $m$ is a set function of $x$. Indeed, let the sets $0, M, V$ be defined by

$$
\varepsilon(x, 0)=0, \quad \varepsilon(x, M)=\frac{1}{2}, \quad \varepsilon(x, V)=1 .
$$

Then $m=0$ for all $x$ for which $\varepsilon(x, x)=0 ; m=M$ for all $x$ such that $\varepsilon(x, x)=\frac{1}{2}$; and $m=V$ for the $x$ for which $\varepsilon(x, x)=1$.

Now, let us look at the proposition

$$
\min _{z}(\varepsilon(z, m) \rightarrow \bar{\varepsilon}(z, m))
$$


which, according to what we have just proved, belongs to our theory. Since $\varepsilon(z, m)=\varepsilon(x, x)$, the proposition can be written

$$
\min _{z}(\varepsilon(x, x) \rightarrow \bar{\varepsilon}(x, x))
$$

which clearly is the same as

$$
\varepsilon(x, x) \rightarrow \bar{\varepsilon}(x, x) .
$$

In virtue of the axiom of comprehension there exists a $y$ such that for all $x$

$$
\varepsilon(x, y)=(\varepsilon(x, x) \rightarrow \bar{\varepsilon}(x, x)) .
$$

In particular it follows that

$$
\varepsilon(y, y)=(\varepsilon(y, y \rightarrow \bar{\varepsilon}(y, y)) .
$$

This, however, is a contradiction because $\varepsilon(y, y)=0, \frac{1}{2}, 1$ are all impossible.

Therefore, we can not use relative quantification in a completely general way to define propositional functions in the axiom of comprehension. A suitable restriction may yield consistency, but how is not known. One might therefore ask if it could be possible to develop mathematics using only the absolute quantification. I do not know whether this is possible or not. An investigation concerning this must be postponed to a later occasion.

The reader will notice that I have not even proved the consistency of the theory with only absolute quantification. It might be inconsistent as well, but that appeares to me improbable.

Assuming that our theory is consistent when only absolute quantification is allowed, I will at last set up a table giving the values of $\varepsilon(x, y)$ for some sets with particularly simple definitions. Let $O, \dot{O}, \ddot{O}, V, \dot{V}, \dot{V}$, $W, \bar{W}$ be defined as follows

$$
\begin{gathered}
\varepsilon(x, O)=0, \quad \varepsilon(x, \dot{O})=\varepsilon(x, x), \\
\varepsilon(x, \ddot{O})=\max _{y}(\min (\varepsilon(x, y), \varepsilon(y, x))), \\
\varepsilon(x, V)=1, \quad \varepsilon(x, \dot{V})=1-\varepsilon(x, x), \\
\varepsilon(x, \ddot{V})=\min _{y}(\max (1-\varepsilon(x, y), 1-\varepsilon(y, x))), \\
\varepsilon(x, W)=\max (\varepsilon(x, x), 1-\varepsilon(x, x)), \\
\varepsilon(x, \bar{W})=\min (\varepsilon(x, x), 1-\varepsilon(x, x)) .
\end{gathered}
$$

The $\varepsilon$-values for the pairs of these 8 sets are given in the table below. 


\begin{tabular}{c|c|c|c|c|c|c|c|c|} 
& $O$ & $\dot{O}$ & $\ddot{O}$ & $V$ & $\dot{V}$ & $\ddot{V}$ & $W$ & $\bar{W}$ \\
\hline$O$ & 0 & 0 & 0 & 1 & 1 & 1 & 1 & 0 \\
\hline$\dot{O}$ & 0 & $\frac{1}{2}$ & 1 & 1 & $\frac{1}{2}$ & 0 & $\frac{1}{2}$ & $\frac{1}{2}$ \\
\hline$\ddot{O}$ & 0 & 1 & 1 & 1 & 0 & 0 & 1 & 0 \\
\hline$V$ & 0 & 1 & 1 & 1 & 0 & 0 & 1 & 0 \\
\hline$\dot{V}$ & 0 & $\frac{1}{2}$ & 1 & 1 & $\frac{1}{2}$ & 0 & $\frac{1}{2}$ & $\frac{1}{2}$ \\
\hline$\ddot{V}$ & 0 & $\frac{1}{2}$ & $\frac{1}{2}$ & 1 & $\frac{1}{2}$ & $\frac{1}{2}$ & $\frac{1}{2}$ & $\frac{1}{2}$ \\
\hline$W$ & 0 & $\frac{1}{2}$ & 1 & 1 & $\frac{1}{2}$ & 0 & $\frac{1}{2}$ & $\frac{1}{2}$ \\
\hline $\bar{W}$ & 0 & $\frac{1}{2}$ & $\frac{1}{2}$ & 1 & $\frac{1}{2}$ & $\frac{1}{2}$ & $\frac{1}{2}$ & $\frac{1}{2}$ \\
\hline
\end{tabular}

\section{REFERENCE}

1. Th. Skolem, Bemerkungen zum Komprehensionsaxiom, Z. Math. Logik Grundlagen Math. 3 (1957), 1-17.

UNIVERSITY OF OSLO, NORWAY 